\title{
PHASED ARRAY ANTENNA FOR THE MITIGATION OF UAS INTERFERENCE
}

\author{
James M. Downey \\ Bryan L. Schoenholz \\ Marie T. Piasecki \\ Robert J. Kerczewski \\ NASA Glenn Research Center \\ Cleveland, Ohio, USA
}

\section{Abstract}

The growing demand for Unmanned Aerial Systems (UAS) operating beyond the line of sight (BLOS) has resulted in an increased interest in using existing commercial satellite communication capabilities for UAS command and control (C2) communications. The World Radiocommunication Conference in 2015 designated portions of $\mathrm{Ku}-\mathrm{Band}$ and Ka-Band fixed satellite service (FSS) spectrum to support UAS C2 communications, provided that potential interference with existing co-allocated users in these bands is addressed. As the user base in this new spectrum allocation expands, there is an increased potential for interference with existing terrestrial communication systems operating under fixed service (FS) allocations. The portion of Ka-Band spectrum allocated for UAS C2 avoids significant interference issues, but the Ku-Band allocation contains a coprimary FS allocation, creating potential interference problems. Therefore, UAS must identify solutions to avoid interfering with these existing FS ground sites while maintaining good links with satellite constellations. UAS operating with conventional fixed feed parabolic antennas will have difficulty in meeting interference thresholds, especially at high latitudes where the antennas will operate with low elevation angles. As a means of addressing this limitation, NASA is investigating the use of a phased array antenna to enable mitigation of interference into ground-based FS receivers. In this paper, a novel lightweight conformal phased array antenna will be presented that can use null-steering and/or beam shaping to avoid ground interference while simultaneously providing strong satellite microwave links for communications. The reduced weight of this design and ability to integrate into the fuselage of smaller UAS platforms will also be discussed as a potential solution to provide BLOS operation via spectrum sharing for an expanding user base. This paper will review design aspects of the conformal phased array antenna, describe the intended benefits in reducing interference with FS ground stations, and describe phased array development and test plans.

\section{Introduction}

As more applications for civil use of UAS continue to be identified and developed, obstacles to routine operation of UAS in the National Airspace (NAS), in particular in non-segregated airspace, are being addressed through research and development, testing, demonstration, and standards development. These obstacles include the development of air traffic management procedures for UAS, validated senseand-avoid systems, and validated command and control (C2) communication systems.

For C2, many UAS applications can make use of line-of-sight (LOS) links to terrestrial ground stations. The range of these types of terrestrial CNPC systems can be extended by networking several terrestrial ground stations to cover a larger geographic area. Prototypes of such systems have been undergoing testing and evaluation and Minimum Operational Performance Standards for terrestrial C2 systems have been developed and published. [1,2]. For UA operations covering large distances or over remote locations, or in areas without adequate terrestrial system coverage, a beyond-line-of-sight (BLOS) CNPC link implemented through a satellite will almost always be required. In addition, some operations requiring redundant $\mathrm{C} 2$ systems to meet availability requirements may include both a terrestrial and a satellite $\mathrm{C} 2$ component.

The International Civil Aviation Organization (ICAO) has determined that the $\mathrm{C} 2$ link providing 
UAS operations in controlled (non-segregated) airspace must use protected aviation safety spectrum. For aeronautical satellite communications links an Aeronautical Mobile Satellite (Route) Service (AMS(R)S) allocation would fulfill this requirement. However, studies on the amount of spectrum required to support UAS BLOS C2 concluded that existing allocations were inadequate.

The International Telecommunications Union (ITU) 2015 World Radiocommunication Conference (WRC-15) considered the use of existing satellites of the Fixed Satellite Service (FSS), of which many operate in several bands, to provide BLOS C2. In particular, the use of FSS in $\mathrm{Ku}-\mathrm{Band}(12-18 \mathrm{GHz})$ and Ka-Band (26.5-40.0 GHz) for BLOS CNPC was considered, and a provisional allocation created. WRC-19 will consider some specific details of these allocations, including protection of existing services from possible interference created by users of the new allocation.

Although the details regarding interference protection are still being debated, in at least some portions of the newly allocated bands the interference protection thresholds will be very difficult to meet, owing to the range of operating characteristics and environments for UAS missions. Approaches to enabling UAS operations while meeting the interference requirements are being studied. The simplest approach consists of limiting UAS operations by imposing minimum altitudes of operation, restricting high latitude operations, and imposing limitations on UAS density.

Such solutions are less than ideal as they place significant restrictions on UAS operations. As an alternative, it is proposed to make use of the beamforming and null-steering capabilities of phased array antennas to dynamically reduce the interference created by UAS C2 satellite transmitters and enable interference protection thresholds to be met under all operating circumstances. As traditional antenna systems can present size and weight challenges for UAS which are generally smaller and lighter than typical commercial aircraft, technology solutions to enable phased array antenna use on UAS are sought.

The NASA Glenn Research Center is leading an effort under NASA's Convergent Aeronautics Solutions (CAS) Program to develop and demonstrate a novel, lightweight, conformal phased array antenna design that could address the interference problem and enable unrestricted UAS operations under satellitebased BLOS C2 link conditions.

The CAS project Conformal Lightweight Antenna Structures for Aeronautical Communications Technologies (CLAS-ACT) is developing and testing antenna technologies that can provide high isotropic effective radiated power (EIRP) in the satellite direction and ultra-low sidelobes in ground direction to enable effective C2 UAS links while minimizing potential interference with terrestrial systems. The antennas will be made with polymer aerogel as a substrate to both reduce weight and improve performance. The aerogel material will also allow antennas to be conformal to the aircraft fuselage to reduce drag.

A prototype subarray will be realized and characterized, including laboratory testing and flight testing on a typical UAS platform. Full system performance will be extrapolated from these results to study the effectiveness of this solution in mitigating UAS satellite-based BLOS C2 link interference with terrestrial systems.

The following sections will describe aspects of satellite communications applied to UAS C2 links and related regulatory aspects. A description of the CLASACT Project will be followed by details of the phased array antenna design and development as well as completed and planned testing activities.

\section{Satellite Communications for BLOS UAS C2 Links}

To provide reliable $\mathrm{C} 2$ communications, terrestrial systems in which the UAS communicates to transmit/receive stations on the ground located within radio line-of-sight (LOS) are generally preferred due to lower cost, smaller and lower weight on-board electronics, and ease of implementation. A number of ground stations can be networked to provide terrestrial system coverage over an area larger than that which can be serviced by a single ground station.

There will be many geographic locations where terrestrial services will not be available due to remoteness and insufficient density of UAS to justify the cost of establishing coverage. Of course, oceanic regions will not have terrestrial coverage either. Even in less remote regions with higher UAS density there may be gaps in coverage. Finally, some UA operations 


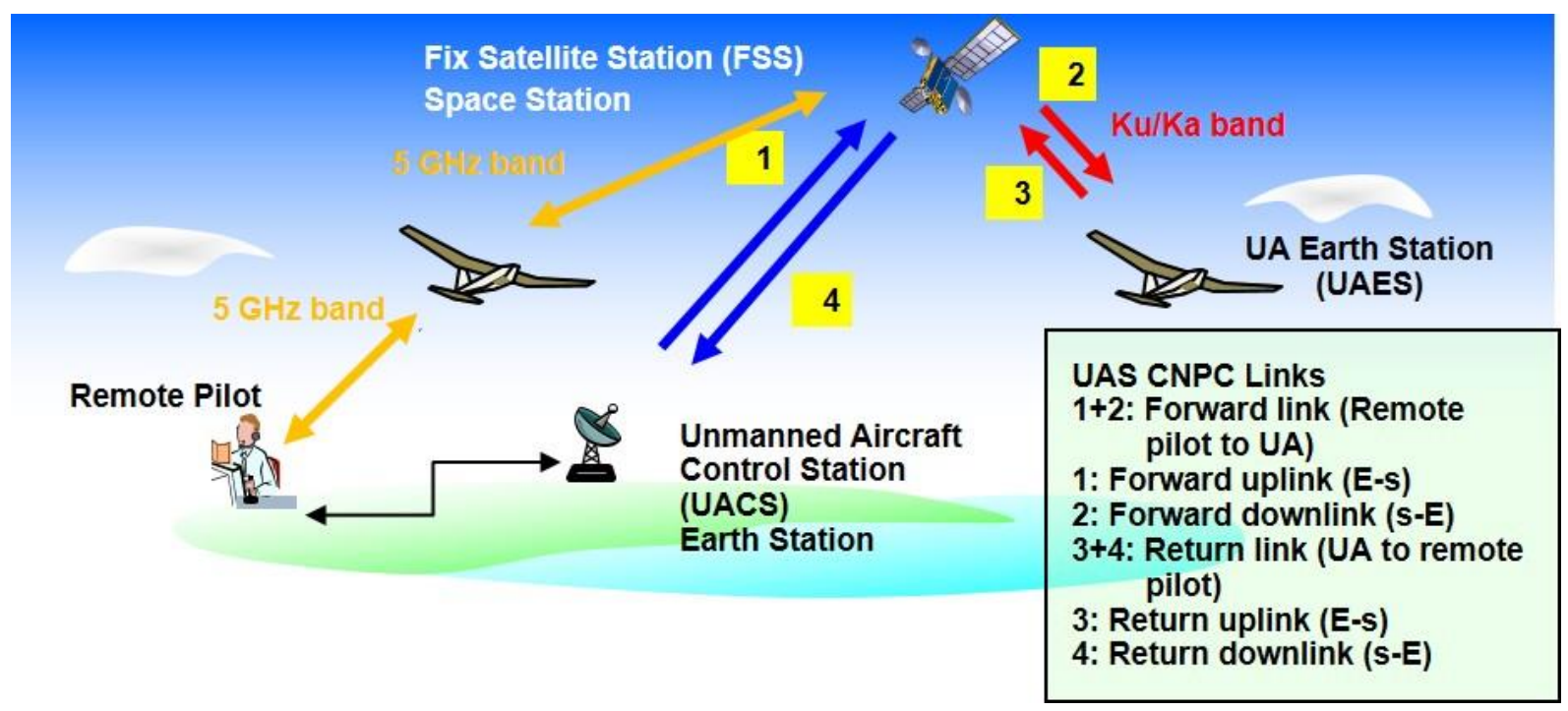

Figure 1. Unmanned Aircraft Systems and Command and Control Links [3].

may require a level of $\mathrm{C} 2$ link availability that makes it necessary to have redundant, independent C2 links consisting of a terrestrial and a satellite component.

Figure 1 [3] shows the $\mathrm{C} 2$ link coverage concept. Terrestrial links directly from the remote pilot operating the UAS, either within radio LOS or through a network of terrestrial stations will make use of an Aeronautical Mobile (Route) Service (AM(R)S) allocation in the $5 \mathrm{GHz}$ band $(5030-5091 \mathrm{GHz})$. Satellite links can be implemented in the same band due to a co-primary AMS(R)S allocation; however, no satellite currently operates in this band. Satellite links can also be operated in two FSS bands in the $\mathrm{Ku}$ - and Ka-Bands. The specific bands are described in the next sections.

Because there are a number of commercial satellite networks operating in both $\mathrm{Ku}$ - and $\mathrm{Ka}-\mathrm{Band}$, the specification of spectrum allocations to specifically provide UAS C2 service was undertaken by WRC-15. This action now makes it possible to make use of commercial FSS systems provided that various technical and operational requirements are satisfied.

\section{Regulatory Aspects of Satellite UAS BLOS C2 Communications}

Spectrum requirements have been established for line-of-sight (LOS) and BLOS CNPC [4]. Actions taken at the 2012 WRC have established spectrum resources to address the LOS spectrum requirements by creating an $\mathrm{AM}(\mathrm{R}) \mathrm{S}$ allocation in the 5030-5091 $\mathrm{MHz}$ band. As noted, this band also has a co-primary AMS(R)S allocation, but no satellite currently operates in this band so satellite-based UAS C2 links are unrealizable

To consider the use of existing satellite services, WRC-15 agenda item 1.5 (AI 1.5) considered "the use of frequency bands allocated to the fixed-satellite service not subject to Appendices 30, 30A and 30B for the control and non-payload communications of unmanned aircraft systems (UAS) in non-segregated airspaces...."[5].

ICAO has determined that $\mathrm{C} 2$ links are aviation safety links and are required to be operated using aviation safety spectrum. However, the FSS is normally not recognized in the ITU as a safety service. Although state-operated UAS have been using C2 links over FSS in segregated airspace for a number of years, for non-segregated airspace additional conditions are needed to meet an equivalent level of safety. ICAO identified the required conditions in the ICAO Position for WRC-15.

Taking into account the ICAO conditions, WRC15 was able to come to an agreement to make new allocations in the FSS $\mathrm{Ku}$ and $\mathrm{Ka}$ frequency bands, identifying over $2.2 \mathrm{GHz}$ of spectrum in WRC-15 Resolution 155. The Resolution specifies that these frequency bands can be used for the UAS C2 links in non-segregated airspace and any other airspace under the control of civil aviation authorities. This use is contingent on the successful development of ICAO 
Standards and Recommended Practices (SARPs). The Resolution goes into considerable detail to protect the current FSS environment against being disrupted by the introduction of a safety service. While not providing a traditional aeronautical mobile satellite (route) service (AMS(R)S), the Resolution introduces an application with safety of life implications into the FSS and contains language consistent with a safety service and appropriately referencing article 4.10 of the ITU Radio Regulations. Further, the Resolution requires ICAO to report on its progress in the development SARPs for the UAS C2 link to WRC-19 and WRC-23, including identification of any problems in the application of the Resolution and potential means by the WRC to address those. The Resolution comes fully into force by WRC-23.

\section{Table 1. WRC-15 Allocations for UAS C2 in the Fixed Satellite Service.}

\begin{tabular}{|l|l|l|}
\hline Band & Space-to-earth & \multirow{2}{*}{ Earth-to-space } \\
\hline \multirow{4}{*}{$\begin{array}{l}\text { Ku- } \\
\text { Band }\end{array}$} & $10.95-11.2 \mathrm{GHz}$ & \multirow{2}{*}{$14-14.47 \mathrm{GHz}$} \\
\cline { 2 - 2 } & $11.45-11.7 \mathrm{GHz}$ & \\
\cline { 2 - 3 } & $11.7-12.2 \mathrm{GHz}$ (ITU Region 2) & \\
\cline { 2 - 3 } $\begin{array}{l}\text { Ka- } \\
\text { Band }\end{array}$ & $12.5-12.75 \mathrm{GHz}$ (ITU Region 1,3) & \\
\hline
\end{tabular}

Table 1 shows the allocations for UAS C2 assigned in Resolution 155. In all of the $\mathrm{Ku}$ Band allocations there are co-primary Fixed Service (FS) allocations covering at least some portions of these allocations in all or some of the ITU Regions. The Fixed Service provides point-to-point and point-tomultipoint terrestrial wireless links. Resolution 155 requires that UAS must accept interference caused by these terrestrial FS systems, and cannot cause harmful interference to these terrestrial FS systems. The Resolution further requires the development of power flux density (PFD) limits that would be applied to UAS C2 transmissions to assure that FS terrestrial receiver will be protected from harmful interference.

Figure 2 describes the UAS-FS interference scenario. Emissions from the UAS transmission to the satellite (link 3 in Figure 2) can create interference into the FS terrestrial receiver (link $3 \mathrm{~s}$ in Figure 2). In addition, the FS terrestrial transmitters can create

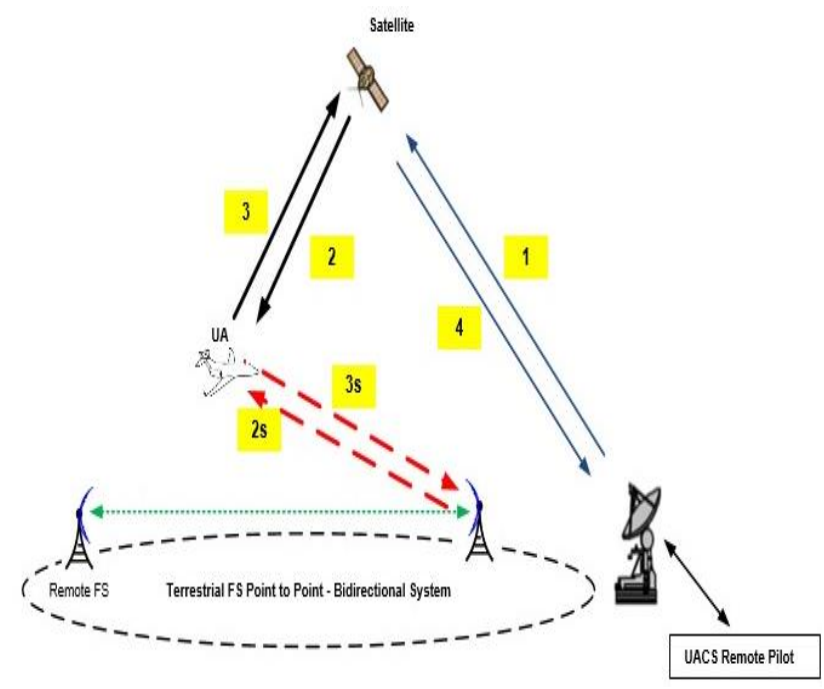

Figure 2. Interference Scenario for UAS C2 Links and Fixed Service Systems.

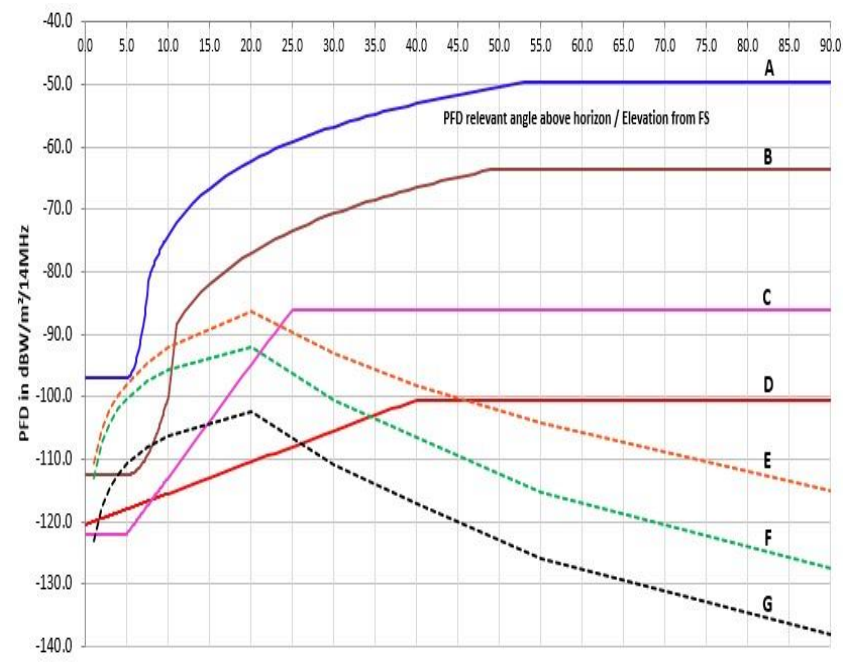

Figure 3. Proposed Power Flux Density Limits Compared to Projected UAS C2 Satellite Transmitter Emissions Using Conventional Antennas. Lines A, B, C and D Represent PFD Limit Proposals. Lines E, F and $G$ are Calculated PFD from UAS Transmitter.

interference into the UAS receiver (link $2 \mathrm{~S}$ in Figure 2). A phased array based solution to reduce the UAS interference into FS receivers can also be applied to reduce the interference the UAS receives from the FS transmitters. 
The PFD limits to be adopted under Resolution 155 will be finalized at WRC-19. Figure 3 shows several proposed PFD limits compared to calculated PFD emissions. The curves labeled A and B in Figure 3 were proposed during the study period leading up to WRC-15[6]. Curve $C$ is a PFD limit proposed during the current WRC-19 study period[3]. Curve D is a PFD limit from an existing ITU-R recommendation that has been used as an example but is not considered applicable to the UAS case [7]. The curves labelled E, $\mathrm{F}$, and $\mathrm{G}$ are calculated PFD emissions for a UAS at 51 degrees latitude using conventional ITU-R standard antenna systems. Curves E and F assume a $3000 \mathrm{ft}$. altitude above ground level (AGL), while curve G assumes $10000 \mathrm{ft}$. altitude [3]. Fuselage attenuation is included in the calculation. This attenuation factor does not have a significant impact below incident angles of 20 degrees. Depending on the PFD limit applied and the antenna characteristics and operating conditions of the UAS, the most difficult conditions in which to avoid UAS interference into FS receivers occur at low indecent angles.

\section{CAS CLAS-ACT Project Description}

The CLAS-ACT project is developing a new lightweight conformal phased array antenna for UAS to help address the interference issues discussed above. Phased array antenna technology can use electronic weighting on each element to form antenna patterns that are otherwise difficult to realize with traditional antenna designs [8]. As shown in Figure 3, traditional dish antennas have difficulty meeting the proposed PFD limits at low elevation angles. The black line in Figure 4, a complementary view of Figure 3 , illustrates the PFD limit line B projected onto an aircraft flying at $51^{\circ} \mathrm{N}$ with an altitude of $3 \mathrm{k}$ feet using an ITU S.465-5 antenna mask (0.8 $\mathrm{m}$ diameter dish, $49.9 \mathrm{dBm}$ EIRP). The red line of Figure 4 shows a simulated pattern for a conformal phased array with similar gain $(\sim 40 \mathrm{dBi})$ to explore the potential for using electronic weighting to aid in meeting PFD limits. The simulated array consists of $49 \times 49$ patch antenna elements placed on a cylindrical surface with a radius of 16". A beam synthesis technique (see method of alternating projections in [9]) was used to show that a synthesized pattern approaches the desired mask, performing $\sim 30 \mathrm{~dB}$ better than an S.465-5 antenna in the $90^{\circ}-100^{\circ}$ region of the pattern. Note that the simulated pattern does not account for fuselage attenuation nor quantization of the electronic weights.

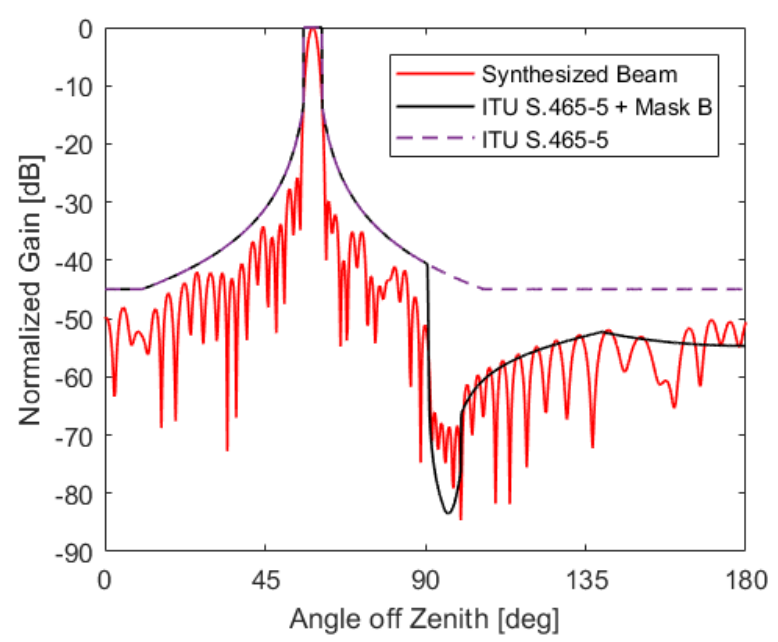

Figure 4. Antenna Mask Requirements Compared to Synthesized Phased Array Pattern

In addition to meeting the ITU mask, another challenge for wider spread usage of satellite C2 communication services is the required volume $(\sim 1$ $\mathrm{m}^{3}$ ) and weight of traditional gimballed satellite dishes. The CLAS-ACT project is attempting to address this by lowering the volume and weight consumed by the satcom (satellite communications) antenna. To achieve this the project is developing a novel flexible polyimide aerogel as the substrate for the antenna structure. Aerogels are $>90 \%$ air and therefore have the potential to significantly reduce the weight of the phased array as well as potentially improve bandwidth or gain of the antenna elements [10]. The conformal nature of the array and lack of a gimbal removes the need for a large open cavity. Instead, the antenna array can be integrated as part of the surface of an aerodynamic airframe.

\section{Lightweight Conformal Phased Array Development}

A sub-scale, 64 element prototype phased array is under development to explore the potential of flexible polyimide aerogels and phased array technology to address regulatory and SWaP (size weight and power) considerations for satcom UAS C2 service. Although a fraction of the size required for satellite communication, 64 elements is expected to be sufficient to demonstrate capability and scalability. Figure 5 shows a possible mounting configuration of the CLAS-ACT prototype array for testing on a UAS. 


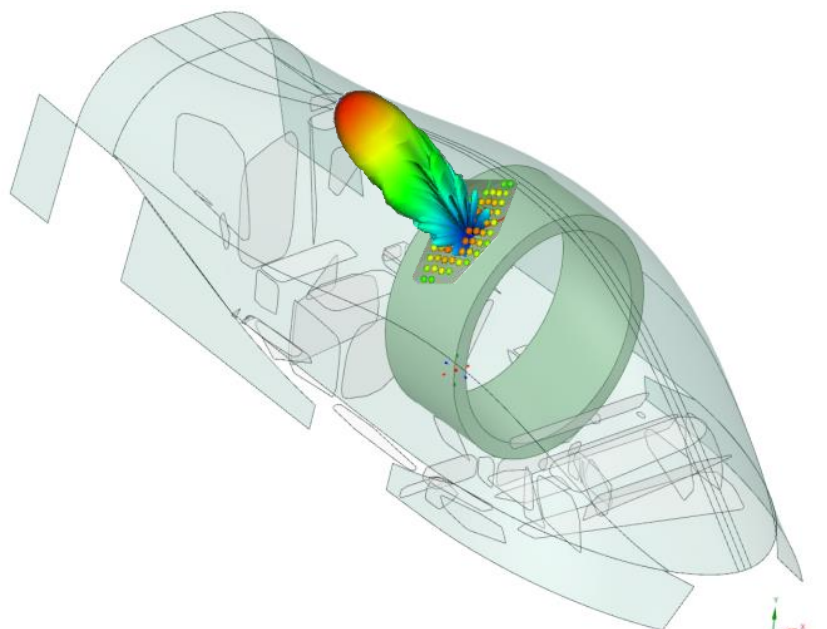

Figure 5. Rendering of 64 Element Prototype Antenna on UAS.

The prototype antenna is a composite of a relatively thick flexible aerogel layer $(\sim 2 \mathrm{~mm})$ for supporting the radiating patch elements and a thin $(<1 \mathrm{~mm})$ multilayer feed stackup made of high performance Rogers microwave substrates (Figure 6). This arrangement maximizes the benefits of the low-dielectric constant aerogel material to support efficient radiation while using the higher dielectric constant materials for the feed network of the array. Compared to using a traditional material for the radiating layer (e.g. PTFE), the aerogel layer results in up to 50\% mass savings for a single radiating layer. Stacked patch configurations for wider bandwidth may see an even larger mass savings. Electronic weighting of each element is achieved using commercially available transmit/receive (TR) chip modules.

Shown in Figure 7, a four element test array was built to verify simulation fidelity and fabrication techniques. The test array made use of a technique to

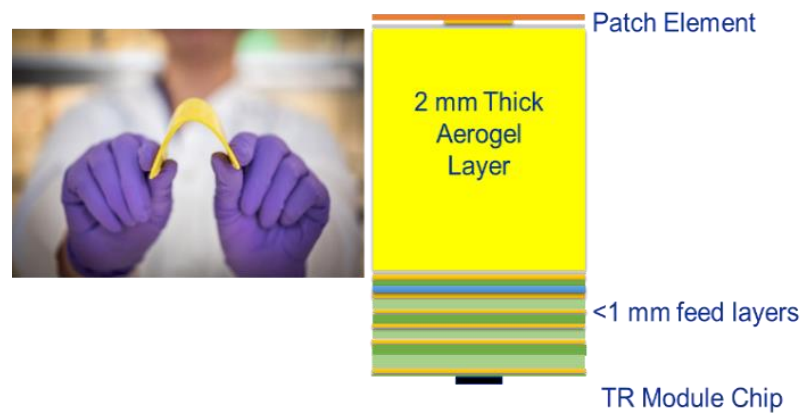

Figure 6. Flexible Polyimide Aerogel (Left). Cross Section of Aerogel Multi-Layer Antenna Stack-Up (Right). align and bond the aerogel substrate with the radiating elements as well as a microstrip feed layer. This array is currently undergoing testing in an anechoic chamber at NASA Glenn Research Center.

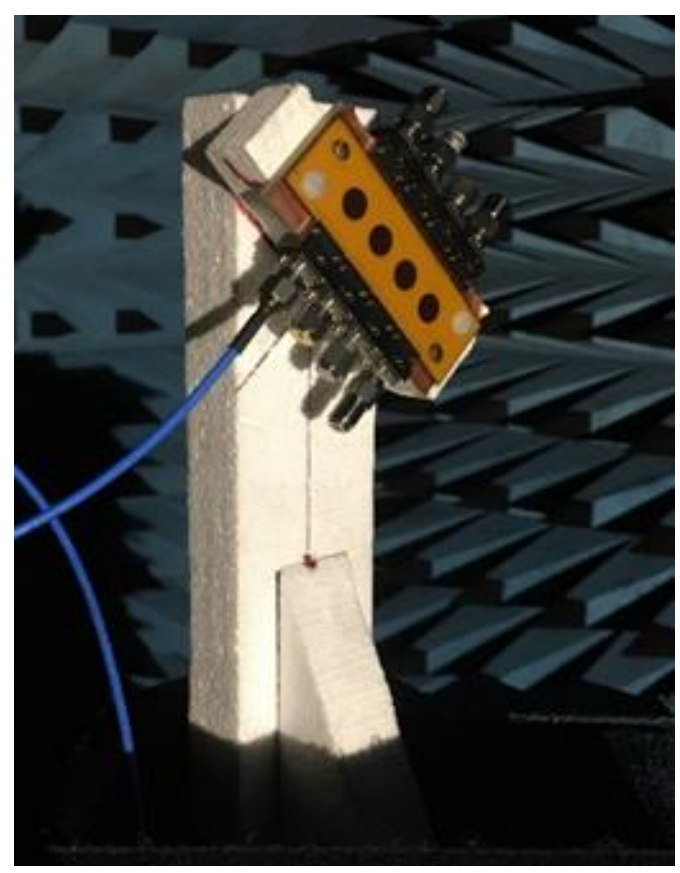

Figure 7. Four Element Array Tested in Anechoic Chamber to Validate Simulation and Fabrication Methods.

\section{Testing of the CLAS-ACT Prototype Subarray}

Evaluating this technology includes three distinct methodologies: antenna chamber testing, on-aircraft ground testing and finally flight testing of prototypes on a UAS. First, the 64 element prototype array will be tested in traditional antenna test chambers located at the NASA Glenn Research Center. These measurements will capture the expected performance of the array including gain and beam steering pattern.

Second, a portable system is under development for ground measurements of the prototype antenna as it is mounted in the UAS. This system, shown in Figure 8 , features a robotic arm mounted on a mobile base along with a laser tracker for precise positioning around a device under test. Setup can be done in an 


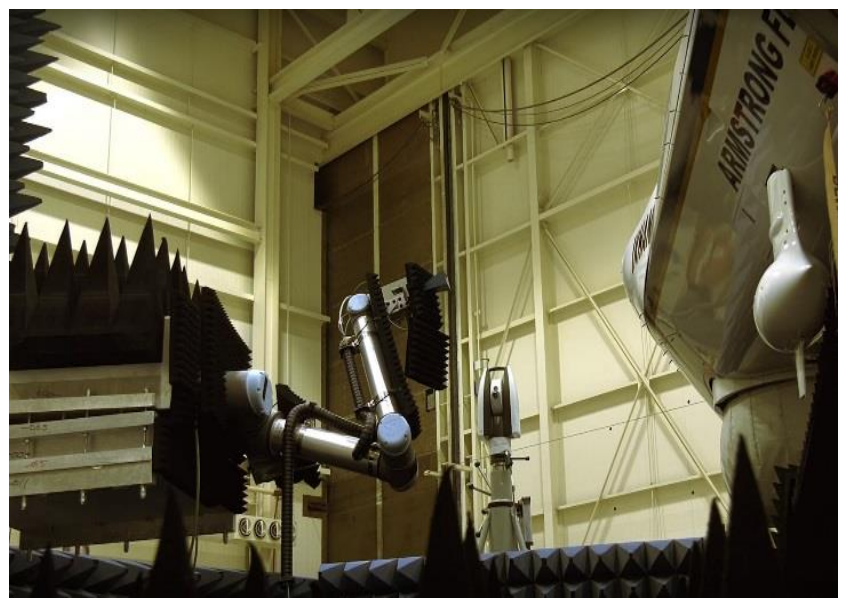

Figure 8. Robotic Arm Based Antenna Pattern Characterization of NASA's Ikhana.

airplane hangar in a day and will allow better fidelity to the data collected in the antenna chambers. In addition to capturing in-situ antenna performance, this measurement system can also be used to measure characteristics of the aircraft itself such as the radome attenuation performance and pattern effects due to the fuselage at a variety of locations around the UAS.

Finally, a flight test will require a measurement ground station (MGS), currently under development, to capture antenna array performance and ground interference in the region of interest during a UAS flight. To accomplish this, an aircraft with the antenna under test will fly various paths past an MGS such that a pattern is measured for a desired angular resolution. This is achieved by varying both the altitude and distance from the MGS for each flight pass. Figure 9 shows an example potential flight pattern. The purpose

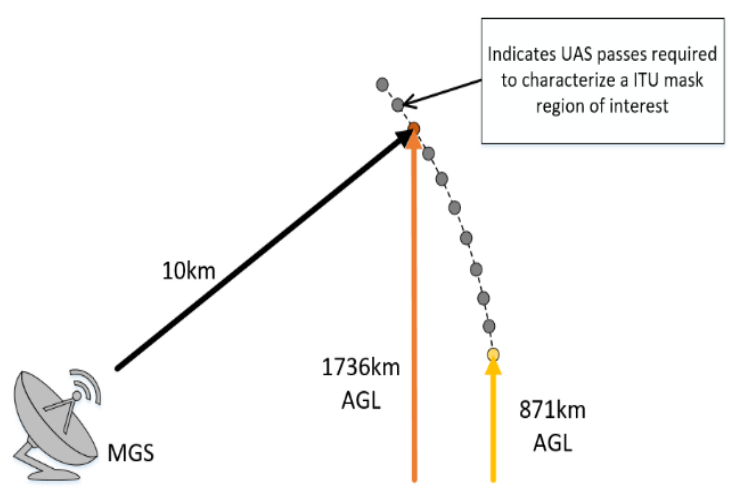

Figure 9. Example Flight Passes for Measuring a Region of the Antenna Pattern. of these three testing systems is to provide a progression of information shown in Figure 10 (Boxes in blue are simulated, green represents anechoic chamber testing, orange is portable robotic scanner, and yellow represents flight testing). By capturing the 64 element array performance in traditional antenna chambers and aircraft characteristics using the robotic arm, an expected performance model can be created. This model is used to predict the installed antenna performance and can be compared against the test of the installed 64 element array on the aircraft in the hanger using the robotic arm. This installed performance data is then used to determine requirements for the MGS which is needed for the final flight testing. The data collected during the flight testing will give the necessary information to make a feasibility assessment for the conformal phased array technology. Overall, this test flow creates a strong level of traceability for performance at any stage. Furthermore, by characterizing the aircraft separately from the array it is possible to decouple any further insitu measurements from the vehicle and offer insight into performance on a variety of UAS.

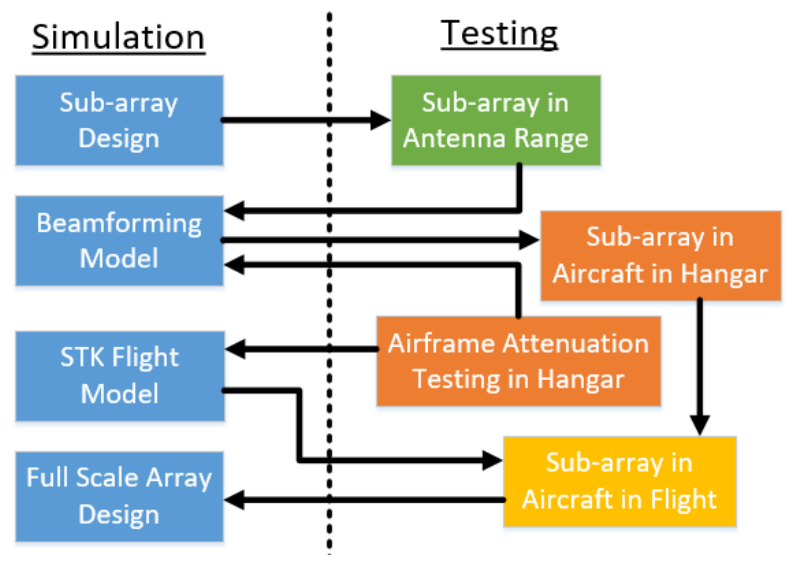

Figure 10. Array Simulation and Testing Flow
Diagram.

\section{Summary}

The use of Ku-band satcom links for UAS C2 communications has been approved by the ITU pending the definition of operational parameters including PFD limits on UAS transmissions. These links can enable growth in operational coverage area while using UAS in segregated airspace, however the challenges identified regarding interference to current 
FS systems on the ground resulting from the expected PFD limits must be addressed. A novel conformal phased array antenna is under development to operate these links while exploiting beam synthesis and null steering techniques to reduce ground interference to acceptable levels. The antenna design will also leverage the use of an ultra-lightweight aerogel material to provide a high performance and low SWaP solution. This low SWaP design may enable smaller UAS to gain BLOS coverage.

\section{Acknowledgements}

The authors wish to thank Dr. Mary Ann Meador's aerogel team and Liz McQuaid of NASA Glenn Research Center, Dr. Kevin Lambert of Vantage Partners LLC, and the NASA Armstrong Flight Research Center Ikhana Ops crew for their contributions to this effort.

The authors also thank William D. Bishop of Jacobs Engineering for graphics developed for this paper.

\section{References}

[1] Shalkhauser, K. A., J. H. Griner and Robert J. Kerczewski, September, 2013, "Flight tests of first generation prototype CNPC radio", Information Paper 06, ICAO Aeronautical Communications Panel, 29th Meeting of Working Group F, Nairobi, Kenya, September 2013, http://legacy.icao.int/anb/panels/acp/wgdoclist.cfm? MeetingID $=296$

[2] RTCA DO-362 - "Command and Control (C2) Data Link Minimum Operational Performance Standards (MOPS) (Terrestrial)", RTCA Inc., 22 September 2016.

[3] ITU-R Document 5B/411E, Report on the Twentieth Meeting of Working Party 5B (Geneva, 6-
17 November 2017), Annex 18 "Working Document Towards a Preliminary Draft New Report ITU-R S.[UA_PFD] - Characteristics of Unmanned Aircraft System Control and Non-Payload Earth Stations for use with Space Stations operating in the Fixed Satellite Service."

[4] ITU-R Report M.2171 - Characteristics of unmanned aircraft systems and spectrum requirements to support safe operation in non-segregated airspace.

[5] Kerczewski, R. J. and L. Jonasson, "Outcomes of the 2015 World Radiocommunication Conference for Aeronautical Spectrum and Applications", 2016 ICNS Conference, Herndon, VA, USA, April 2016.

[6] Kerczewski, R., Wilson, J., Bishop, W., “Analysis of Terrestrial Interference Protection from UAS CNPC Satellite Transmitters", 2016 ICNS Conference, Herndon, VA, USA, April 2016.

[7] "Technical and operational requirements for aircraft earth stations of aeronautical mobile-satellite service including those using fixed-satellite service network transponders in the band 14-14.5 GHz (Earthto-space)", ITU-R Recommendation M.1643

[8] Brookner, E., January 2006, "Phased Arrays and Radars - Past Present and Future," Microwave Journal.

[9] Josefsson, L. and P. Persson, 2006 "Conformal Array Antenna Theory and Design," Hoboken, New Jersey, Wiley.

[10] Meador, M. A., F. A. Miranda, 2014 "Design and Development of Aerogel-Based Antennas for Aerospace Applications: A Final Report to the NARI Seedling," NASA/TM - 2014-218346.

\section{Integrated Communications Navigation and Surveillance (ICNS) Conference} April 10-12, 2018 